\title{
Distribuição geográfica e análise morfológica de Artibeus lituratus Olfers e de Artibeus fimbriatus Gray (Chiroptera, Phyllostomidae) no Rio Grande do Sul, Brasil
}

\author{
Ana Maria Rui ${ }^{1}$ \\ Marta Elena Fabián ${ }^{1}$ \\ João Oldair Menegheti ${ }^{1}$
}

\begin{abstract}
Geographical distribution and morphological analysis of Artibeus lituratus Olfers and Artibeus fimbriatus Gray (Chiroptera, Phyllostomidae) in Rio Grande do Sul, Brazil. A study has been made on the geographical distribution and comparative external and cranial morphological analysis of Artibeus lituratus Olfers, 1818 and Artibeus fimbriatus Gray, 1838 (Chiroptera, Phyllostomidae) in the state of Rio Grande do Sul, Brazil. A. lituratus and A. fimbriatus were found to be sympatric in the state north to the "Planície Costeira", in the "Depressão Central", in the hillsides of "Serra Geral", and in the northern region of the state, areas previously covered by forests. The southernmost point for Artibeus Leach, 1821 distribution is $30^{\circ}$ South, and A. lituratus and A. fimbriatus are the two species found more to the south. The two species studied do not show sexual dimorphism as to external characteristics. Cranial measurements revealed significant differences between males and females of $A$. lituratus in mandible length, which was significantly larger in females( $<<5 \%)$, and between males and females of $A$. fimbriatus, in the length of the set of lower teeth and in the external width between the cingula of canine teeth, which were significantly larger in males $(\mathrm{p}<5 \%)$. No further morphological cranial differences were found between genders of both species. A. lituratus and A. fimbriatus can be externally distinguished by size, for $A$. lituratus is larger than A. fimbriatus as concerns all external dimensions analysed except for the tibia length $(\mathrm{p}<5 \%)$. The two species can also be differentiated by pelage colour, hair length, and facial stripes appearance. In the skull, a number of differences were found in rostrum format, in the developmental degree of supraorbital and post-orbital crests and pre-orbital and post-orbital processes, and in several cranial dimensions analysed.

KEY WORDS. Phyllostomidae, Artibeus lituratus, Artibeus fimbriatus, distribution, morphology, South Brazil
\end{abstract}

Os morcegos do gênero Artibeus Leach, 1821 (Phyllostomidae, Stenodermatinae) distribuem-se desde o México até o norte da Argentina e sul do Brasil (JONES \& CARTER 1976; KOOPMAN 1982; MARQUES-AGUIAR 1994).

Atualmente são reconhecidas oito espécies de grandes Artibeus (subgênero Artibeus): Artibens amplus Handley, 1987, Artibeus fimbriatus Gray, 1838, Artibeus fraterculus Anthony, 1924, Artibeus hirsutus Andersen, 1906, Artibeus inopinatus Davis \& Carter, 1964, Artibeus jamaicensis Leach, 1821, Artibeus lituratus Olfers, 1818 e Artibeus obscurus Schinz, 1821 (HANDLEY 1987, 1989, 1991; MARQUES-AGUIAR 1994). Alguns autores reconhecem ainda a existência de mais

1) Departamento de Zoologia, Instituto de Biociências, Universidade Federal do Rio Grande do Sul. Avenida Paulo Gama, 90040-060 Porto Alegre, Rio Grande do Sul, Brasil. 
duas espécies, $A$. intermedius Allen, 1897 para a América Central (DAvIS 1984) e A. planirostris (Spix, 1823) para a América do Sul (KoOPMAN 1982; OwEN 1987).

Artibeus lituratus e A. fimbriatus são espécies convergentes morfologicamente em diversos aspectos e foram confundidas durante muito tempo ao longo de suas áreas de ocorrências (HANDLEY 1989). Porém, recentemente alguns autores trabalharam com as duas espécies diferenciando-as e esclarecendo a posição taxonômica de A. fimbriatus. Entre esses autores, MYERS \& WETZEL (1979, 1983), registraram a presença de $A$. fimbriatus para a parte oriental do Paraguai e realizaram uma análise morfológica comparativa das três espécies que ocorrem naquela região. HANDLEY (1989) redescreveu A. fimbriatus diferenciando-o das demais espécies, além de fornecer informações sobre sua distribuição geográfica.

A. lituratus ocorre desde a parte central do México até o sul do Brasil, estado do Rio Grande do Sul, e no norte da Argentina, estando presente também nas Pequenas Antilhas e em Trinidade e Tobago (JONES \& CARTER 1976; KoOPMAN 1982; MARQUES-AGUIAR 1994). No Brasil A. fimbriatus há registros para os estados da Bahia, Rio de Janeiro, São Paulo, Paraná e Santa Catarina (MARQuES-AGUIAR 1994), ocorre também no leste do Paraguai (MYers \& WeTZEL 1979, 1983) e em algumas províncias do norte da Argentina (BARQUEZ \& OJEDA 1992).

O presente trabalho tem como objetivo registrar a ocorrência de A. fimbriatus no Rio Grande do Sul, ampliando sua área de distribuição, e fornecer dados sobre a distribuição geográfica de $A$. lituratus e de $A$. fimbriatus no Estado. É apresentada também uma análise morfológica das características externas e cranianas das duas espécies com o objetivo de verificar a existência de variações individuais, dimorfismo sexual e determinar as características diferenciais entre $A$. lituratus e $A$. fimbriatus no sul do Brasil.

\section{MATERIAL E MÉTODOS}

Os dados sobre a distribuição geográfica de $A$. lituratus e de $A$. fimbriatus no Rio Grande do Sul foram obtidos através de exame de coleções científicas e de revisão bibliográfica.

As coleções científicas examinadas são as seguintes: Laboratório de Mastozoologia do Museu de Ciências e Tecnologia (MZMCT) da Pontifícia Universidade Católica do Rio Grande do Sul (PUCRS), Museu de Ciências Naturais (MCN) da Fundação Zoobotânica do Rio Grande do Sul (FZBRS) e coleção do Laboratório de Mastozoologia do Departamento de Zoologia (DZMAM) da Universidade Federal do Estado do Rio Grande do Sul (UFRGS).

Material examinado. Artibeus lituratus, BRAsIL, Rio Grande do Sul: Derrubadas, machos: MCN 2411, MCN 2421, MCN 2430, MCN 2433, MCN 2434, MCN 2436, MCN 2438, MCN 2446, MCN 2447, MCN 2449, MCN 2470, MCN 2486, MCN 2488, MCN 2491; fêmeas: MCN 2414, MCN 2415, MCN 2416, MCN 2417, MCN 2418, MCN 2429, MCN 2431, MCN 2435, MCN 2440, MCN 2441, MCN 2442, MCN 2444, MCN 2445, MCN 2448, MCN 2451, MCN 2453, MCN 2472, MCN 2473, MCN 2487, MCN 2489, MCN 2490; Dom Pedro de Alcantâra, macho: DZMAM 0171; fêmea: DZMAM 0172; Encantado, macho: MCN 554; General Câmara, fêmea: MCN 281; Maquiné, machos: DZMAM 12, DZMAM 19, DZMAM 
20, DZMAM 25, DZMAM 33, DZMAM 34, DZMAM 60, DZMAM 64, DZMAM 65, DZMAM 66, DZMAM 67, DZMAM 68, DZMAM 75, DZMAM 76, DZMAM 77, DZMAM 88, DZMAM 89, DZMAM 91, DZMAM 92, DZMAM 99; fêmeas: DZMAM 09, DZMAM 10, DZMAM 16, DZMAM 21, DZMAM 26, DZMAM 53, DZMAM 59, DZMAM 70, DZMAM 71, DZMAM 72, DZMAM 73, DZMAM 74, DZMAM 86, DZMAM 90, DZMAM 93, DZMAM 94, DZMAM 95, DZMAM 96, DZMAM 97, DZMAM 98, DZMAM 100; Porto Alegre, macho: DZMAM 201; Santa Maria, fêmea: MCN 288; Torres, machos: MCN 417, MCN 535; fêmeas: MCN 418, MCN 420, MCN 534. Artibeus fimbriatus, BRAsIL, Rio Grande do Sul: Derrubadas, machos: MCN 2403, MCN 2454; fêmeas: MCN 2443, MCN 2469, MCN 2471; Dom Pedro de Alcantâra, macho: DZMAM 0173; fêmea: DZMAM 0174; Maquiné, machos: DZMAM 15, DZMAM 23, DZMAM 24, DZMAM 35, DZMAM 37, DZMAM 49, DZMAM 52; fêmeas: DZMAM 08, DZMAM 14, DZMAM 18, DZMAM 22, DZMAM 40, DZMAM 41, DZMAM 42, DZMAM 43, DZMAM 45, DZMAM 47, DZMAM 48; Porto Alegre, machos: MCTMZ 0281, MCN 51, MCN 841; Restinga Seca, machos: MCN 949, MCN 950; fêmeas: MCN 946, MCN 948; Santa Cruz do Sul, fêmeas: MCN 211, MCN 212; Santa Maria, fêmea: MCN 287; São Francisco de Paula, fêmea: MCN 748; Taquara, macho: MCN 815; Torres, fêmea: MCN 536; Triunfo, macho: MCN 2369.

Para a análise morfométrica foram obtidas medidas externas e do crânio de exemplares das duas espécies com um paquímetro Mitutoyo de $150 \mathrm{~mm}$ e precisão de $0,05 \mathrm{~mm}$. Todas as medidas foram baseadas em VIzOTTO \& TADDEI (1973).

Foram calculados para cada grupo de medidas a média e o desvio padrão. Para a análise estatística das medidas foram aplicados o teste F, para comparação entre variâncias, e o teste t, para comparação entre médias.

Compararam-se as medidas das dimensões externas e cranianas de machos e fêmeas de cada espécie para determinar a existência ou não de dimorfismo sexual. Diante de diferenças significativas entre os sexos, machos e fêmeas foram considerados separadamente. Quando inexistiram diferenças significativas, as medidas de machos e fêmeas foram reunidas em um só grupo, para a comparação entre as duas espécies. dows ${ }^{\circledR} 3.11$.

O aplicativo estatístico usado foi Microsoft Excel $^{\circledR} 5.0$ em ambiente Win-

\section{RESULTADOS E DISCUSSÃO}

\section{Distribuição Geográfica}

A ocorrência de Artibeus lituratus no Rio Grande do Sul foi registrada anteriormente para várias localidades. VIEIRA (1942) e TADDEI (1979) referem-se a IHERING (1895) que registrou a espécie no município de Porto Alegre. SANTOS (1978) coletou exemplares em São Leopoldo e em Panambi. WALLAUER \& ALBUQUERQUE (1986) registraram a ocorrência de A. lituratus no Parque Estadual do Turvo, extremo norte do Estado. HANDLEy (1989) cita a espécie para Santo Cristo, no noroeste do Estado. A espécie está presente também em Nonoai, no Parque Estadual de Nonoai, norte do Estado. Artibeus fimbriatus não havia sido registrado anteriormente no Estado. 
Artibeus lituratus e A. fimbriatus estão distribuídos no Rio Grande do Sul no norte da Planície Costeira até a altura do município de Porto Alegre, para oeste na Depressão Central e encostas da Serra Geral até a altura do município de Santa Maria e também no norte do Estado (Fig. 1).

Comparando-se a distribuição das duas espécies no Rio Grande do Sul, constata-se que suas áreas de ocorrência praticamente se sobrepõem (Fig. 1). Dados de literatura também mostram que as duas espécies são simpátricas em outras regiões e que A. lituratus é a espécie mais comum e a mais abundante (HANDLEY 1989).

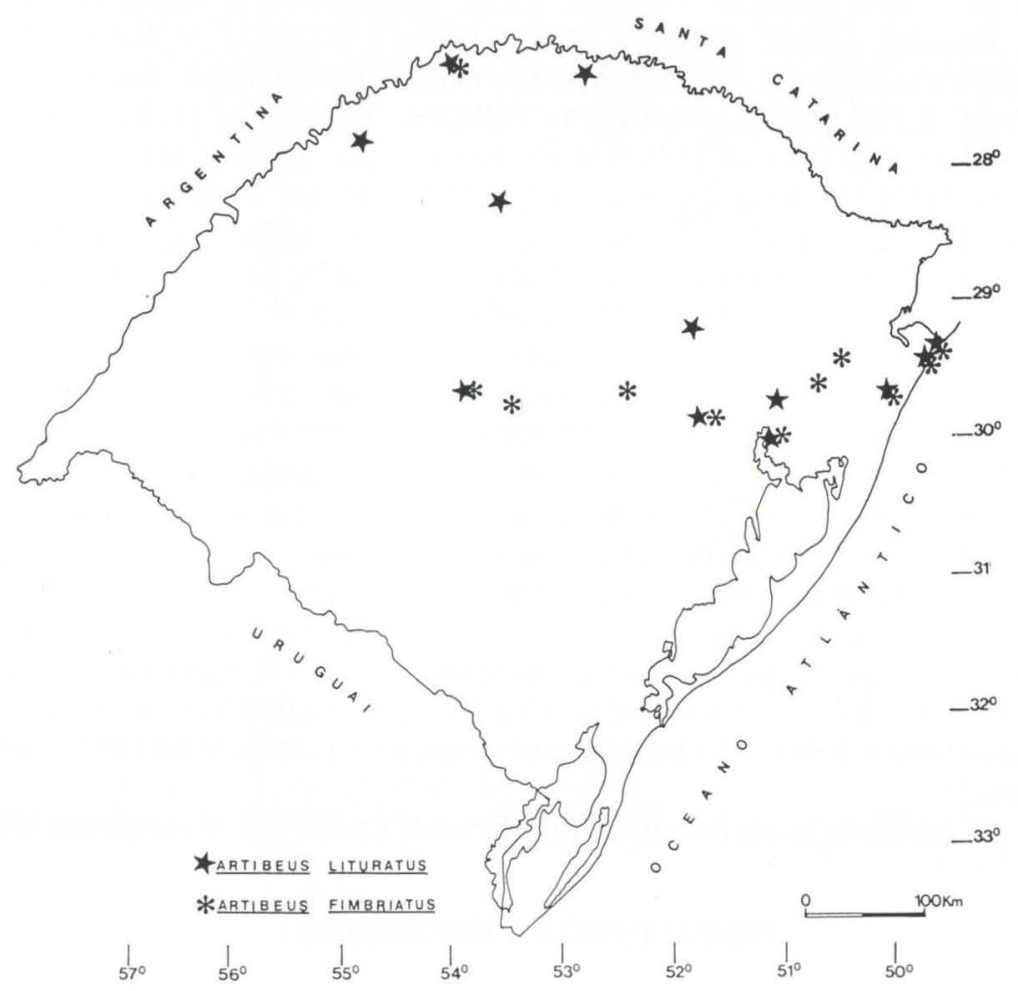

Fig. 1. Distribuição geográfica de Artibeus lituratus e de Artibeus fimbriatus no Rio Grande do Sul, Brasil.

No Rio Grande do Sul a distribuição geográfica de A. lituratus e de $A$. fimbriatus está relacionada com áreas originalmente cobertas por florestas. As duas espécies estão distribuídas em regiões de ocorrência da Floresta Ombrófila Densa, na Planície Costeira. Para oeste, na Depressão Central e nas encostas da Serra Geral, em regiões de Floresta Estacional Semidecidual e de Floresta Estacional Decidual. Foram registradas também no norte do Estado nas grandes áreas florestais do Parque 
Estadual do Turvo e do Parque Estadual de Nonoai, na Mata Pluvial do Alto Uruguai, em Floresta Estacional Decidual. É provável também que Artibeus ocorra, abaixo de $30^{\circ} \mathrm{S}$ de latitude, na parte leste do Planalto-Sul-Rio-Grandense onde originalmente havia uma grande extensão de Floresta Estacional Semidecidual (RADAM/BRASIL 1986). Porém, não há trabalhos disponíveis sobre a fauna de Chiroptera desta região. Inexistem registros da presença de $A$. lituratus e $A$. fimbriatus nas regiões de domínio das demais formações vegetais do Estado.

O limite meridional de distribuição do gênero Artibeus no Brasil é o estado do Rio Grande do Sul em torno de $30^{\circ} \mathrm{S}$ de latitude, sendo que as duas espécies de distribuição mais ao sul são $A$. lituratus e $A$. fimbriatus. Já na Argentina as espécies do gênero ( $A$. lituratus, A. fimbriatus, $A$. jamaicensis e $A$. planirostris) foram registradas nas províncias de Salta, Tucumán, Misiones, Chaco e Formosa, norte do país (BARQUEZ \& OJEDA 1992; REDFORD \& EISENBERG 1992; MARQUES-AGUIAR 1994).

\section{Morfologia Externa}

Artibeus lituratus é a maior espécie do gênero Artibeus encontrada no Rio Grande do Sul, com comprimento médio de antebraço de $69,08 \mathrm{~mm} \pm 1,876$ $(65,30-72,00)$ (Tab. I). Os exemplares examinados apresentam cor marrom escuro. A parte ventral do corpo é sempre mais clara que a dorsal. Na face há a presença de duas listras brancas, largas e bem delimitadas que se estendem da região da folha nasal até as orelhas. A folha nasal apresenta a borda inferior da ferradura fusionada medianamente ao lábio e bordas laterais livres. As asas são da mesma cor do corpo do animal e suas extremidades podem ser brancas, mais claras que as asas ou da mesma cor da asa. A membrana interfemoral é larga e moderadamente peluda, com pelagem mais densa na parte dorsal.

Tabela I. Média $(\bar{X})$, desvio padrão (DP) e niveis de significância dos testes te $\mathrm{F}$ das medidas externas de Artibeus lituratus (machos e fêmeas) e Artibeus fimbriatus (machos e fêmeas) provenientes de diversas regiöes do Rio Grande do Sul, Brasil. (n) Número de indivíduos.

\begin{tabular}{|c|c|c|c|c|c|c|}
\hline \multirow[t]{2}{*}{ Medidas externas } & \multicolumn{2}{|c|}{ Artibeus literatus $(n=30)$} & \multicolumn{2}{|c|}{ Artibeus fimbriatus $(n=30)$} & \multirow{2}{*}{$\begin{array}{l}\text { Niveis de } \\
\text { significância } \\
\text { do Teste t }\end{array}$} & \multirow{2}{*}{$\begin{array}{c}\text { Niveis de } \\
\text { significância } \\
\text { do Teste F }\end{array}$} \\
\hline & $\bar{x}$ & DP & $\bar{x}$ & DP & & \\
\hline Antebraço & 69,08 & 1,876 & 63,98 & 1,680 & $5,54-16$ & 0,5570 \\
\hline Orelha & 22,94 & 2,252 & 21,14 & 1,070 & 0,0002 & 0,0001 \\
\hline Trago & 8,56 & 0,611 & 7,85 & 0,540 & $1,48 \mathrm{E}-05$ & 0,5140 \\
\hline Tibia & 25,93 & 1,479 & 25,34 & 1,112 & 0,0850 & 0,1300 \\
\hline Terceiro metacarpo & 66,08 & 1,934 & 59,19 & 1,738 & $5,80 E-21$ & 0,5680 \\
\hline Primeira falange do $3^{\circ}$ dedo & 23,70 & 0,966 & 20,79 & 1,277 & $4,17 \mathrm{E}-14$ & 0,1380 \\
\hline Segunda falange do $3^{\circ}$ dedo & 37,58 & 1,554 & 33,69 & 1,205 & $1,45 \mathrm{E}-15$ & 0,1760 \\
\hline Terceira falange do $3^{\circ}$ dedo & 20,02 & 1,236 & 18,62 & 1,003 & $1,09 \mathrm{E}-05$ & 0,2650 \\
\hline Quarto metacarpo & 63,76 & 2,088 & 57,44 & 1,601 & $4,90 \mathrm{E}-19$ & 0,1580 \\
\hline Primeira falange do $4^{\circ}$ dedo & 19,85 & 0,837 & 18,02 & 1,180 & $3,74 \mathrm{E}-09$ & 0,0680 \\
\hline Segunda falange do $4^{\circ}$ dedo & 24,08 & 1,322 & 22,10 & 1,435 & $6,80 \mathrm{E}-20$ & 0,6620 \\
\hline Quinto metacarpo & 65,92 & 2,180 & 58,55 & 1,827 & $1,64 \mathrm{E}-20$ & 0,3470 \\
\hline Primeira falange do $5^{\circ}$ dedo & 15,50 & 0,833 & 14,11 & 1,091 & $7,79 E-07$ & 0,1510 \\
\hline Segunda falange do $5^{\circ}$ dedo & 19,18 & 1,068 & 17,11 & 1,310 & $8,65 E-09$ & 0,2760 \\
\hline Calcâneo & 8,30 & 0,698 & 6,85 & 0,508 & $6,17 \mathrm{E}-13$ & 0,0920 \\
\hline
\end{tabular}


Artibeus fimbriatus é menor que $A$. lituratus, com comprimento médio do antebraço de $63,98 \mathrm{~mm} \pm 1,680(59,30-67,35)$ (Tab. I). A coloração da pelagem é cinza fuliginosa, bastante escura na parte dorsal do corpo. Ventralmente a pelagem é mais clara, com a extremidade distal dos pêlos branca, o que dá um aspecto esbranquiçado a esta região do corpo. A face, a folha nasal, as orelhas, as asas e o uropatágio são cinzentos, quase pretos. As listras faciais são fracamente delimitadas, estreitas, um pouco mais claras que a pelagem dos animais. O pêlo da parte dorsal do corpo é longo e sedoso. A folha nasal possui a borda inferior da ferradura soldada medianamente ao lábio e extremidades laterais livres, com bordas geralmente onduladas. As extremidades das asas podem ser brancas, mais claras que a cor das asas ou da mesma cor destas. Os membros posteriores e membrana interfemoral possuem pêlos que são mais abundantes no dorso.

Através do teste $\mathrm{t}$ constatou-se não haver diferenças significativas entre as médias das medidas externas dos machos e das fêmeas de $A$. lituratus e de $A$. fimbriatus para um nível de significância de 5\% (Tabs II, III). Ou seja, não há dimorfismo sexual quanto ao tamanho nestas duas espécies. Também não se constatou outra característica morfológica externa que possa caracterizar dimorfismo sexual nas duas espécies estudadas.

Tabela II. Média $(\bar{X})$, desvio padrão (DP) e niveis de significância dos testes te $F$ das medidas externas de machos e fêmeas de Artibeus lituratus provenientes de diversas regiōes do Rio Grande do Sul, Brasil.

\begin{tabular}{|c|c|c|c|c|c|c|}
\hline \multirow[t]{2}{*}{ Medidas externas } & \multicolumn{2}{|c|}{ Machos $(n=15)$} & \multicolumn{2}{|c|}{ Fèmeas $(n=15)$} & \multirow{2}{*}{$\begin{array}{l}\text { Niveis de } \\
\text { significância } \\
\text { do Teste t }\end{array}$} & \multirow{2}{*}{$\begin{array}{c}\text { Niveis de } \\
\text { significância } \\
\text { do Teste F }\end{array}$} \\
\hline & $\bar{x}$ & DP & $\bar{x}$ & $\mathrm{DP}$ & & \\
\hline Antebraço & 69,02 & 1,447 & 69,15 & 2,277 & 0,860 & 0,101 \\
\hline Orelha & 22,41 & 2,587 & 23,48 & 1,787 & 0,195 & 0,178 \\
\hline Trago & 8,45 & 0,763 & 8,67 & 0,406 & 0,335 & 0,024 \\
\hline Tíbia & 26,07 & 1,063 & 25,80 & 1,834 & 0,626 & 0,050 \\
\hline Terceiro metacarpo & 65,97 & 1,725 & 66,20 & 2,178 & 0,743 & 0,393 \\
\hline Primeira falange do $3^{\circ}$ dedo & 23,60 & 0,972 & 23,80 & 0,983 & 0,579 & 0,964 \\
\hline Segunda falange do $3^{\circ}$ dedo & 37,53 & 1,702 & 37,64 & 1,449 & 0,859 & 0,555 \\
\hline Terceira falange do $3^{\circ}$ dedo & 19,86 & 1,427 & 20,18 & 1,036 & 0,483 & 0,243 \\
\hline Quarto metacarpo & 63,46 & 1,781 & 64,06 & 2,380 & 0.438 & 0,289 \\
\hline Primeira falange do $4^{\circ}$ dedo & 19.79 & 1,015 & 19,92 & 0,641 & 0,678 & 0,097 \\
\hline Segunda falange do $4^{\circ}$ dedo & 23,97 & 1,492 & 24,20 & 1,169 & 0,637 & 0,371 \\
\hline Quinto metacarpo & 65,42 & 1,967 & 66,42 & 2,331 & 0,213 & 0,534 \\
\hline Primeira falange do $5^{\circ}$ dedo & 15,40 & 0,976 & 15,60 & 0,680 & 0,513 & 0,189 \\
\hline Segunda falange do $5^{\circ}$ dedo & 19,10 & 1,325 & 19,26 & 0,771 & 0,696 & 0,051 \\
\hline Calcâneo & 8,17 & 0,762 & 8,44 & 0,625 & 0,297 & 0,468 \\
\hline
\end{tabular}

TADDEI (1979), analisou exemplares de A. lituratus provenientes da região norte-ocidental do estado de São Paulo e encontrou diferenças significativas entre os sexos, com um nível de significância de $5 \%$, no comprimento da terceira falange do terceiro dedo e no comprimento da segunda falange do quinto dedo e, com nível de significância de $1 \%$, no comprimento da cabeça-corpo, comprimento da orelha, comprimento do antebraço, comprimento do terceiro metacarpo, comprimento do quarto metacarpo e comprimento do quinto metacarpo. As diferenças entre os resultados obtidos por esse autor e os do presente trabalho podem ser atribuídas 
provavelmente a variações existentes entre populações de diferentes regiões geográficas. Deve-se considerar também que TADDEI (1979) utilizou em sua análise o dobro $(n=60)$ de exemplares que foram usados no presente trabalho, o que pode ter influenciado nos resultados obtidos.

Tabela III. Média $(\bar{X})$, desvio padrão (DP) e niveis de significância dos testes t e $\mathrm{F}$ das medidas externas de machos e fêmeas de Artibeus fimbriatus provenientes de diversas regiões do Rio Grande do Sul, Brasil. (n) Número de indivíduos.

\begin{tabular}{|c|c|c|c|c|c|c|}
\hline \multirow[t]{2}{*}{ Medidas externas } & \multicolumn{2}{|c|}{ Machos $(n=15)$} & \multicolumn{2}{|c|}{ Fêmeas $(n=15)$} & \multirow{2}{*}{$\begin{array}{c}\text { Niveis de } \\
\text { significância } \\
\text { do Teste t }\end{array}$} & \multirow{2}{*}{$\begin{array}{l}\text { Niveis de } \\
\text { significância } \\
\text { do Teste F }\end{array}$} \\
\hline & $\bar{x}$ & DP & $\bar{x}$ & $\mathrm{DP}$ & & \\
\hline Antebraço & 63,60 & 1,475 & 64,36 & 1,834 & 0,223 & 0,425 \\
\hline Orelha & 21,23 & 0,864 & 21,04 & 1,266 & 0,629 & 0,165 \\
\hline Trago & 7,83 & 0,660 & 7,88 & 0,410 & 0,805 & 0,084 \\
\hline Tibia & 25,27 & 1,107 & 25,42 & 1,150 & 0,724 & 0,889 \\
\hline Terceiro metacarpo & 58,92 & 1,841 & 59,46 & 1,646 & 0,404 & 0,681 \\
\hline Primeira falange do $3^{\circ}$ dedo & 21,15 & 0,817 & 20,44 & 1,561 & 0,130 & 0,021 \\
\hline Segunda falange do $3^{\circ}$ dedo & 33,67 & 1,480 & 33,71 & 0,903 & 0,929 & 0,074 \\
\hline Terceira falange do $3^{\circ}$ dedo & 18,71 & 1,250 & 18,53 & 0,709 & 0,638 & 0,041 \\
\hline Quarto metacarpo & 57,43 & 1,683 & 57,46 & 1,574 & 0,955 & 0,805 \\
\hline Primeira falange do $4^{\circ}$ dedo & 18,31 & 0,989 & 17,73 & 1,314 & 0,180 & 0,299 \\
\hline Segunda falange do $4^{\circ}$ dedo & 21,85 & 1,213 & 22,35 & 1,632 & 0,349 & 0,279 \\
\hline Quinto metacarpo & 58,55 & 1,925 & 58,55 & 1,793 & 0,992 & 0,793 \\
\hline Primeira falange do $5^{\circ}$ dedo & 14,28 & 1,121 & 13,94 & 1,071 & 0,402 & 0,866 \\
\hline Segunda falange do $5^{\circ}$ dedo & 16,90 & 1,074 & 17,32 & 1,520 & 0,389 & 0,206 \\
\hline Calcâneo & 6,96 & 0,373 & 6,75 & 0,610 & 0,273 & 0,076 \\
\hline
\end{tabular}

$\mathrm{Na}$ análise comparativa entre as duas espécies o teste $t$ mostrou que há diferenças significativas entre as médias de todas as medidas externas, com exceção do comprimento da tíbia, para os níveis de significância indicados na tabela I. Artibeus lituratus é maior que A. fimbriatus em relação a todas as medidas das dimensões externas analisadas.

Através da análise morfológica constatou-se que as principais características externas distintivas entre $A$. lituratus e $A$. fimbriatus são o tamanho, a cor da pelagem, o comprimento do pêlo, que é mais longo no dorso de $A$. fimbriatus, e a aparência das listras faciais. Porém, há pequenas variações individuais na cor da pelagem e nitidez das listras faciais em ambas as espécies.

Myers \& Wetzel $(1979,1983)$ afirmam que no Paraguai $A$. lituratus pode ser distinguido externamente de $A$. jamaicensis e $A$. fimbriatus por seu tamanho maior e por possuir listras faciais definidas. HANDLEY (1989) afirma que $A$. lituratus e $A$. fimbriatus podem ser separadas pela cor e comprimento do pêlo, pelo aspecto das listras faciais e pelo tamanho, sendo que $A$. lituratus possui antebraço e dedos mais longos, e as demais dimensões externas similares a $A$. fimbriatus. Os dados obtidos por estes autores a respeito das características diferenciais entre $A$. lituratus e $A$. fimbriatus são similares aos obtidos para as duas espécies no Rio Grande do Sul.

MARQues-Aguiar (1994) afirma que em A. lituratus a folha nasal é livre do lábio, podendo em alguns indivíduos ser fusionada medianamente, e que as extremidades das asas em A. fimbriatus são sempre brancas. Estas duas caracterís- 
ticas divergiram no material examinado proveniente do Rio Grande do Sul. Provavelmente estas diferenças constatadas entre o material examinado por MARQUESAGUIAR (1994) e os do presente trabalho podem ser atribuídas a variações geográficas ao longo da distribuição das duas espécies.

\section{Morfologia Craniana}

Os exemplares de $A$. lituratus examinados apresentam crânio com comprimento total médio de 31,98 mm $\pm 0,753$ (30,25- 33,30) (Tab. IV). O crânio desta espécie apresenta rostro longo e achatado dorsalmente. As cristas supra-orbitais e pós-orbitais são bem desenvolvidas e salientes, assim como os processos pré-orbitais e pós-orbitais, o que dá ao rostro um aspecto de escudo. A constrição pós-orbital é bastante acentuada, com largura média de $6,76 \mathrm{~mm} \pm 0,300(6,25-7,35)$ (Tab. IV). A mandíbula apresenta o processo angular largo e o processo articular não proeminente. A fórmula dentária da espécie é $2 / 2,1 / 1,2 / 2,2 / 3 \times 2=30$ (Fig. 2).

Tabela IV. Média $(\bar{X})$, desvio padrão (DP) e niveis de significância dos testes $t$ e $F$ das dimensões cranianas de Artibeus lituratus (machos e fêmeas) e de Artibeus fimbriatus (machos e fêmeas) provenientes de diversas regiões do Rio Grande do Sul, Brasil. (n) Número de individuos.

\begin{tabular}{|c|c|c|c|c|c|c|}
\hline \multirow[t]{2}{*}{ Medidas do crânio } & \multicolumn{2}{|c|}{$\begin{array}{l}\text { Artibeus literatus } \\
\qquad(n=32)\end{array}$} & \multicolumn{2}{|c|}{$\begin{array}{l}\text { Artibeus fimbriatus } \\
\qquad(n=32)\end{array}$} & \multirow{2}{*}{$\begin{array}{l}\text { Niveis de } \\
\text { significância } \\
\text { do Teste t }\end{array}$} & \multirow{2}{*}{$\begin{array}{l}\text { Niveis de } \\
\text { significância } \\
\text { do Teste F }\end{array}$} \\
\hline & $\bar{x}$ & DP & $\bar{x}$ & $\mathrm{DP}$ & & \\
\hline Comprimento total & 31,98 & 0,753 & 31,51 & 0,617 & 0,009 & 0,275 \\
\hline Comprimento côndilo basal & 28,49 & 0,670 & 27,37 & 0,507 & 0,421 & 0,125 \\
\hline Comprimento basal & 24,91 & 0,638 & 24,77 & 0,514 & 0,363 & 0,233 \\
\hline Comprimento palatal & 15,00 & 0,530 & 15,37 & 0,476 & 0,004 & 0,550 \\
\hline Comprimento da série de dentes superiores & 11,48 & 0,291 & 11,69 & 0,301 & 0,005 & 0,857 \\
\hline Largura externa entre molares & 14,06 & 0,373 & 14,27 & 0,333 & 0,019 & 0,528 \\
\hline Largura inter-orbitária & 8,02 & 0,360 & 8,30 & 0,355 & 0,002 & 0,943 \\
\hline Largura poss-orbitária & 6,76 & 0,300 & 7,60 & 0,249 & $5,26 \mathrm{E}-18$ & 0,303 \\
\hline Largura zigomática & 19,39 & 0,611 & 19,19 & 0,474 & 0,155 & 0,163 \\
\hline Largura da porçăo craniana & 13,78 & 0,360 & 13,36 & 0,312 & $5,55 E-06$ & 0,430 \\
\hline Largura mastóidea & 17,12 & 0,502 & 16,73 & 0,396 & 0,001 & 0,195 \\
\hline Largura palatal & 7,50 & 0,381 & 7,54 & 0,284 & 0,630 & 0,109 \\
\hline Altura do occipital & 9.17 & 0,366 & 9,03 & 0,298 & 0,090 & 0,261 \\
\hline
\end{tabular}

A. fimbriatus apresenta comprimento total médio do crânio de $31,51 \mathrm{~mm} \pm$ 0,617 (30,20-32,70) (Tab. IV). A espécie apresenta rostro longo e arqueado dorsalmente e sem aspecto de escudo. As cristas supra-orbitais e pós-orbitais e os processos pré-orbitais e pós-orbitais não são desenvolvidos ou são pobremente desenvolvidos. A constrição pós-orbital em $A$. fimbriatus é pouco acentuada, com largura média de $7,60 \mathrm{~mm} \pm 0,249(7,00-8,10)$ (Tab. IV). A porção craniana apresenta uma saliência na confluência das cristas pós-orbitais na parte superior do rostro. Na mandíbula o processo angular é estreito e o processo articular é não proeminente. A fórmula dentária da espécie é 2/2, 1/1, 2/2, 2/3 x $2=30$ (Fig. 3).

Quanto ao crânio, as duas espécies apresentam algumas pequenas variações individuais em suas características, que podem ser atribuídas, provavelmente, às diferenças de idade. Estas variações foram observadas principalmente no formato do escudo rostral e no grau de desenvolvimento das cristas supra-orbitais e pós-or- 
bitais e dos processos pré-orbitais e pós-orbitais. As duas espécies possuem a mesma fórmula dentária, que se mostrou constante. HANDLEY (1989), ao referir-se a $A$. fimbriatus, afirma que a espécie apresenta poucas variações morfológicas cranianas e que não foram constatadas variações geográficas nos espécimes por ele examinados.
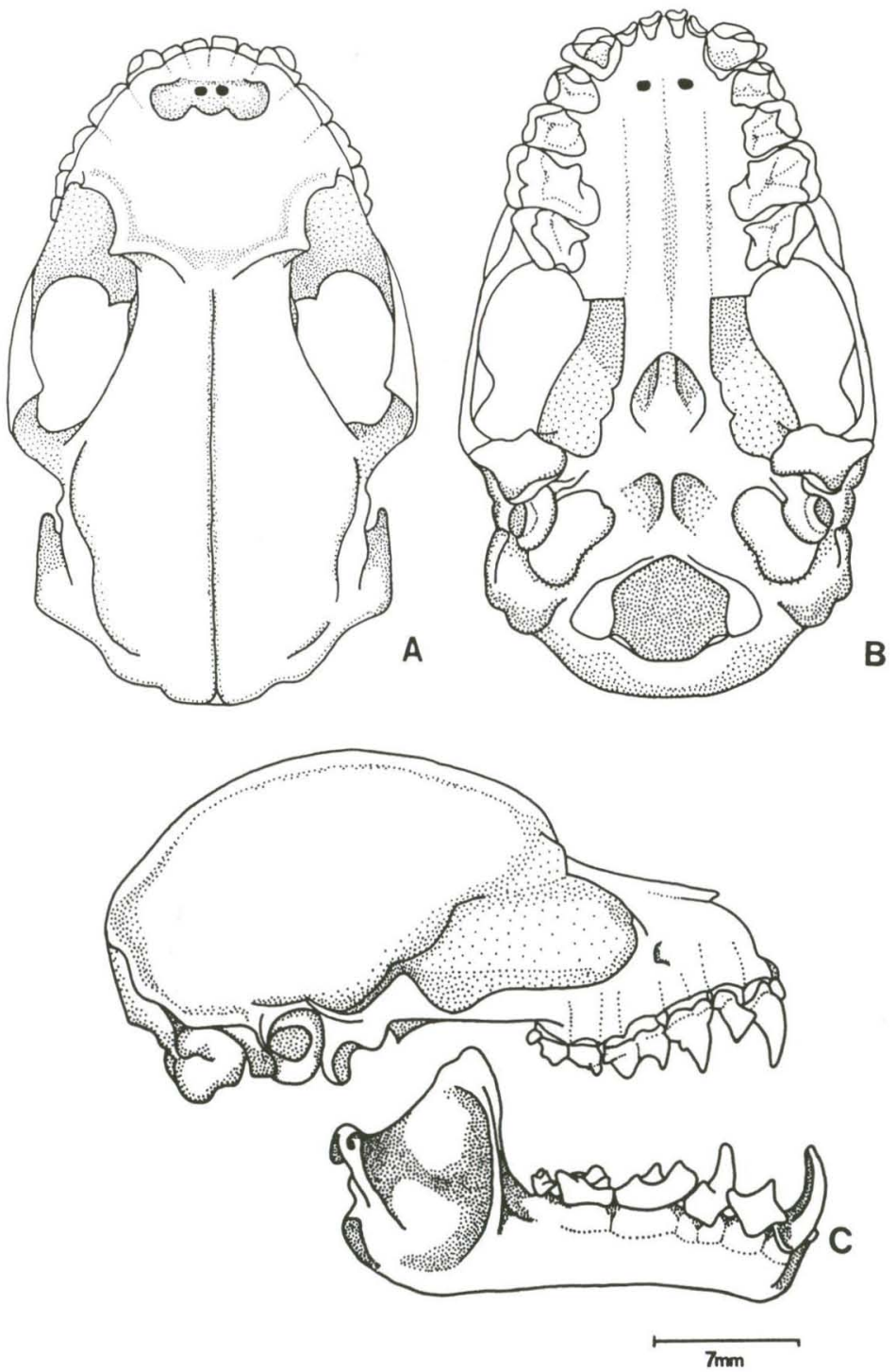

Fig. 2. Crânio de Artibeus lituratus, exemplar DZMAM 96 fêmea, proveniente do municipio de Maquiné (Rio Grande do Sul). (A) Vista dorsal, (B) vista ventral, (C) vista lateral direita. 

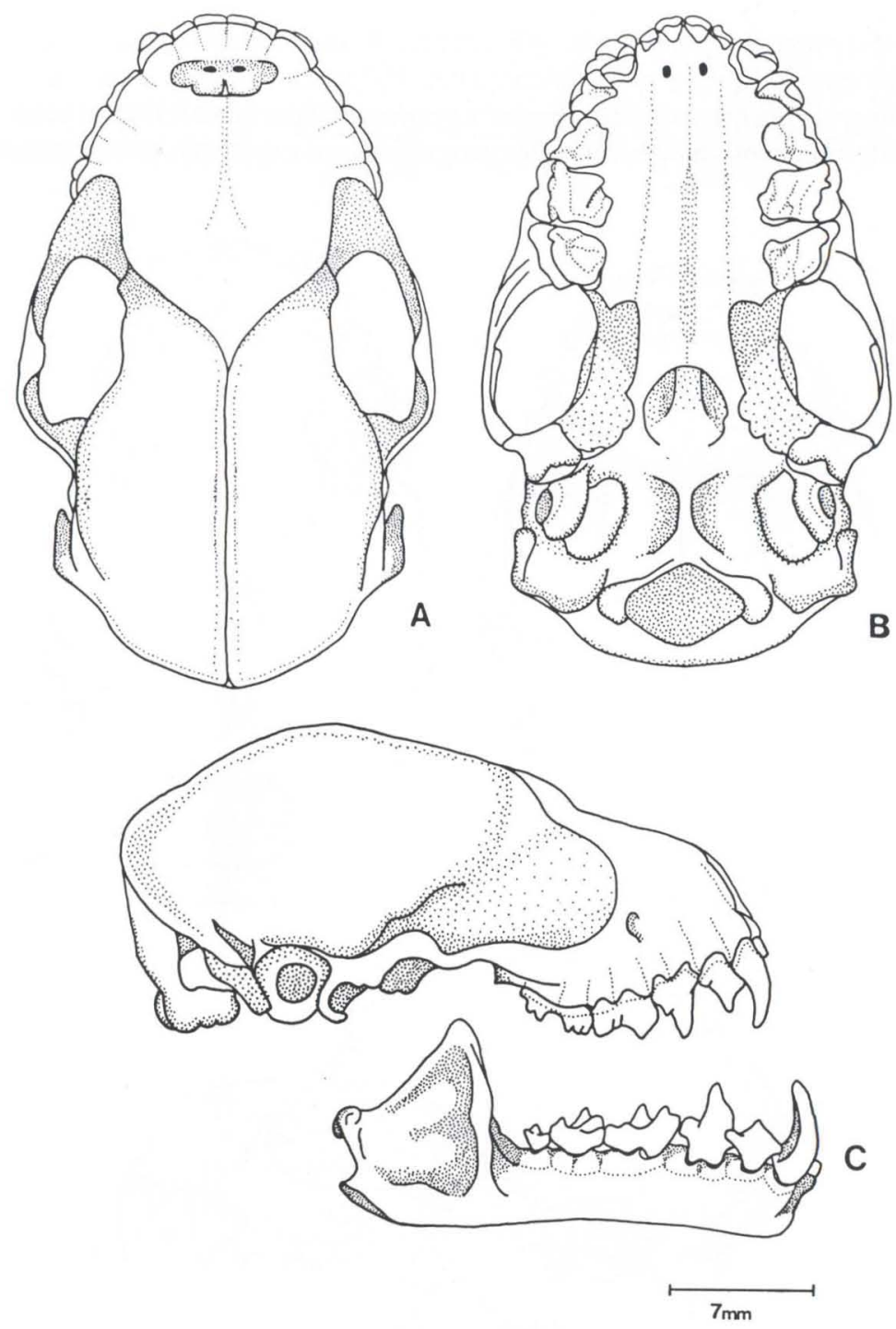

Fig. 3. Crânio de Artibeus fimbriatus, exemplar MCTMZ 0281 macho, proveniente do municipio de Porto Alegre (Rio Grande do Sul). (A) Vista dorsal, (B) vista ventral, (C) vista lateral direita.

Constatou-se não haver diferenças para um nível de significância de $5 \%$ entre as médias das medidas do crânio de machos e fêmeas de $A$. lituratus, com exceção do comprimento da mandíbula, que foi significativamente maior nas fêmeas (Tab. V). Machos e fêmeas de A. fimbriatus apresentaram diferenças para um nível de significância de 5\% nas médias do comprimento da série de dentes inferiores e na 
largura externa entre os cingulas dos caninos, que foram significativamente maiores nos machos (Tab. VI). Não foram constatadas outras características cranianas que evidenciem dimorfismo sexual em A. lituratus e A. fimbriatus.

Tabela V. Média $(\bar{X})$, desvio padrão (DP) e niveis de significância dos testes t e $F$ das dimensões cranianas de machos e fêmeas de Artibeus lituratus provenientes de diversas regiöes do Rio Grande do Sul, Brasil. (n) Número de indivíduos.

\begin{tabular}{|c|c|c|c|c|c|c|}
\hline \multirow[t]{2}{*}{ Medidas do crânio } & \multicolumn{2}{|c|}{ Machos $(n=16)$} & \multicolumn{2}{|c|}{ Fêmeas $(n=16)$} & \multirow{2}{*}{$\begin{array}{c}\text { Niveis de } \\
\text { significância } \\
\text { do Teste t }\end{array}$} & \multirow{2}{*}{$\begin{array}{c}\text { Niveis de } \\
\text { significância } \\
\text { do Teste F }\end{array}$} \\
\hline & $\bar{x}$ & DP & $\bar{x}$ & DP & & \\
\hline Comprimento total & 31,87 & 0,830 & 32,08 & 0,670 & 0,440 & 0,430 \\
\hline Comprimento côndilo basal & 28,37 & 0,746 & 28,61 & 0,581 & 0,305 & 0,344 \\
\hline Comprimento basal & 24,69 & 0,662 & 25,12 & 0,554 & 0,058 & 0,501 \\
\hline Comprimento palatal & 14,93 & 0,610 & 15,06 & 0,447 & 0,503 & 0,240 \\
\hline Comprimento da série de dentes superiores & 11,47 & 0,322 & 11,49 & 0,267 & 0,836 & 0,474 \\
\hline Comprimento da série de dentes inferiores & 12,89 & 0,374 & 13,02 & 0,333 & 0,303 & 0,660 \\
\hline Comprimno da mandibula & 20,39 & 0,498 & 21,80 & 0,727 & 4,47E-07 & 0,153 \\
\hline Largura externa entre as cingulas dos caninos & 9,13 & 0,257 & 9,03 & 0,289 & 0,295 & 0,658 \\
\hline Largura externa entre os molares & 14,11 & 0,403 & 14,01 & 0,347 & 0,486 & 0,568 \\
\hline Largura inter-orbitária & 8,01 & 0,419 & 8,03 & 0,303 & 0,848 & 0,221 \\
\hline Largura pós-orbitária & 6,80 & 0,308 & 6,71 & 0,296 & 0,419 & 0,873 \\
\hline Largura zigomática & 19,38 & 0,764 & 19,40 & 0,433 & 0,921 & 0,035 \\
\hline Largura da porção craniana & 13,76 & 0,388 & 13,79 & 0,341 & 0,848 & 0,630 \\
\hline Largura mastóidea & 17,18 & 0,577 & 17,06 & 0,425 & 0,535 & 0,249 \\
\hline Largura palatal & 7,46 & 0,449 & 7,53 & 0,310 & 0,650 & 0,165 \\
\hline Altura do occipital & 9,17 & 0,318 & 9,17 & 0,419 & 0,962 & 0,294 \\
\hline
\end{tabular}

Tabela VI. Média $(\bar{X})$, desvio padrão (DP) e níveis de significância dos testes t e $F$ das dimensões cranianas de machos e fêmeas de Artibeus fimbriatus provenientes de diversas regiōes do Rio Grande do Sul, Brasil. (n) Número de indivíduos.

\begin{tabular}{|c|c|c|c|c|c|c|}
\hline \multirow[t]{2}{*}{ Medidas do crânio } & \multicolumn{2}{|c|}{ Machos $(n=16)$} & \multicolumn{2}{|c|}{ Fêmeas $(n=16)$} & \multirow{2}{*}{$\begin{array}{l}\text { Niveis de } \\
\text { significância } \\
\text { do Teste t }\end{array}$} & \multirow{2}{*}{$\begin{array}{l}\text { Niveis de } \\
\text { significância } \\
\text { do Teste F }\end{array}$} \\
\hline & $\bar{x}$ & $\mathrm{DP}$ & $\bar{x}$ & DP & & \\
\hline Comprimento total & 31,60 & 0,714 & 31,43 & 0,513 & 0,457 & 0,210 \\
\hline Comprimento cơndilo basal & 28,40 & 0,588 & 28,34 & 0,428 & 0,759 & 0,229 \\
\hline Comprimento basal & 24,77 & 0,598 & 24,78 & 0,434 & 0,986 & 0,228 \\
\hline Comprimento palatal & 15,41 & 0,384 & 15,32 & 0,561 & 0,573 & 0,153 \\
\hline Comprimento da série de dentes superiores & 11,70 & 0,297 & 11,68 & 0,314 & 0,818 & 0,830 \\
\hline Comprimento da série de dentes inferiores & 12,84 & 0,318 & 12,58 & 0,313 & 0,029 & 0,951 \\
\hline Comprimno da mandibula & 20,16 & 0,571 & 20,30 & 0,462 & 0,450 & 0,423 \\
\hline Largura externa entre as cingulas dos caninos & 9,12 & 0,293 & 8,91 & 0,161 & 0,023 & 0,026 \\
\hline Largura externa entre os molares & 14,35 & 0,332 & 14,20 & 0,326 & 0,207 & 0,948 \\
\hline Largura inter-orbitária & 8,27 & 0,382 & 8,33 & 0,336 & 0,627 & 0,621 \\
\hline Largura pós-orbitária & 7,63 & 0,177 & 7,56 & 0,306 & 0,405 & 0,041 \\
\hline Largura zigomática & 19,26 & 0,448 & 19,13 & 0,505 & 0,454 & 0,652 \\
\hline Largura da porçăo craniana & 13,36 & 0,341 & 13,35 & 0,290 & 0,911 & 0,533 \\
\hline Largura mastóidea & 16,78 & 0,376 & 16,69 & 0,424 & 0,541 & 0,648 \\
\hline Largura palatal & 7,55 & 0,263 & 7,52 & 0,311 & 0,715 & 0,520 \\
\hline Altura do occipital & 9,10 & 0,321 & 8,95 & 0,262 & 0,159 & 0,439 \\
\hline
\end{tabular}

TADDEI (1979) não encontrou diferenças significativas entre as dimensões cranianas de machos $(\mathrm{n}=15)$ e fêmeas $(\mathrm{n}=15)$ de A. lituratus coletados na região norte-ocidental do estado de São Paulo. Provavelmente as diferenças nos resultados obtidos por este autor e no presente trabalho podem ser atribuídas a variações geográficas na espécie. 
$\mathrm{Na}$ análise morfométrica craniana entre as duas espécies constatou-se haver diferenças significativas, a um nível de $5 \%$, entre as médias das seguintes dimensões cranianas de $A$. lituratus e $A$. fimbriatus: comprimento total do crânio, comprimento palatal, comprimento da série de dentes superiores, largura externa entre os molares, largura interorbitária, largura pós-orbitária, largura da porção craniana e largura mastóidea (Tab. IV). Verificaram-se também diferenças a um nível de $5 \%$ no comprimento da mandíbula de $A$. fimbriatus (machos e fềmeas) e das fêmeas de $A$. lituratus (Tab. VII); e entre A. lituratus (machos e fêmeas) e as fêmeas de $A$. fimbriatus no comprimento da série de dentes inferiores e na largura externa entre os cingulas dos caninos (Tab. VIII).

Tabela VII. Média $(\bar{X})$ e desvio padrão (DP) do comprimento da mandíbula de Artibeus fimbriatus (machos e fêmeas) e niveis de significância dos testes t e $F$ entre as medidas de Artibeus fimbriatus (machos e fêmeas) e Artibeus lituratus (machos) e entre Artibeus fimbriatus (machos e fêmeas) e Artibeus lituratus (fêmeas). Exemplares provenientes de diversas regiões do Rio Grande do Sul, Brasil. (n) Número de indivíduos.

\begin{tabular}{|c|c|c|c|c|c|c|}
\hline \multirow[t]{2}{*}{ Medidas do crânio } & \multicolumn{2}{|c|}{$\begin{array}{l}\text { A. literatus }(n=32) \\
\text { (machos e Fèmeas) }\end{array}$} & \multicolumn{2}{|c|}{$\begin{array}{l}\text { A. fimbriatus }(n=32) \times \\
\text { A. lituratus machos }(n=16)\end{array}$} & \multicolumn{2}{|c|}{$\begin{array}{l}\text { A. fimbriatus }(n=32) \times \\
\text { A. lituratus fêmeas }(n=16)\end{array}$} \\
\hline & $\bar{x}$ & DP & $\begin{array}{c}\text { Niveis de } \\
\text { significância "t" }\end{array}$ & $\begin{array}{c}\text { Niveis de } \\
\text { significância "F" }\end{array}$ & $\begin{array}{c}\text { Niveis de } \\
\text { significância "t" }\end{array}$ & $\begin{array}{c}\text { Niveis de } \\
\text { significância " } F "\end{array}$ \\
\hline Comprimento da mandibula & 20,23 & 0,516 & 0,317 & 0,913 & $3,42 \mathrm{E}-11$ & 0,104 \\
\hline
\end{tabular}

Tabela VIII. Média $(\bar{X})$ e desvio padrão (DP) de dimensões cranianas de Artibeus lituratus (machos e fêmeas) e níveis de significância dos testes t e F entre as medidas de Artibeus lituratus (machos e fêmeas) e Artibeus fimbriatus (machos) e entre Artibeus lituratus (machos e fêmeas) e Artibeus fimbriatus (fêmeas). Exemplares provenientes de diversas regiões do Rio Grande do Sul, Brasil. (n) Número de indivíduos.

\begin{tabular}{|c|c|c|c|c|c|c|}
\hline \multirow[t]{2}{*}{ Medidas do crånio } & \multicolumn{2}{|c|}{$\begin{array}{l}\text { A. literatus ( } n=32) \\
\text { (machos e Fèmeas) }\end{array}$} & \multicolumn{2}{|c|}{$\begin{array}{l}\text { A. fimbriatus }(n=32) \times \\
\text { A. lituratus machos }(n=16)\end{array}$} & \multicolumn{2}{|c|}{$\begin{array}{l}\text { A. fimbriatus }(n=32) \times \\
\text { A. lituratus fêmeas }(n=16)\end{array}$} \\
\hline & $\bar{x}$ & DP & $\begin{array}{l}\text { Niveis de } \\
\text { significância "t" }\end{array}$ & $\begin{array}{c}\text { Niveis de } \\
\text { "significância "F" }\end{array}$ & $\begin{array}{l}\text { Niveis de } \\
\text { significância " } \mathrm{t} \text { " }\end{array}$ & $\begin{array}{c}\text { Niveis de } \\
\text { significância " } F "\end{array}$ \\
\hline $\begin{array}{l}\text { Comprimento da série de dentes } \\
\text { inferiores }\end{array}$ & 12,95 & 0,355 & 0,290 & 0,675 & 0,0009 & 0,625 \\
\hline $\begin{array}{l}\text { Largura externa entre as cingulas } \\
\text { dos caninos }\end{array}$ & 9,08 & 0,274 & 0,678 & 0,725 & 0,0110 & 0,031 \\
\hline
\end{tabular}

A. fimbriatus apresentou as médias do comprimento palatal, comprimento da série de dentes superiores, largura externa entre os molares, a largura interorbitária e a largura pós-orbitária significativamente maiores do que A. lituratus. As médias do comprimento total do crânio, da largura da porção craniana e da largura mastóidea foram significativamente maiores em $A$. lituratus.

As características cranianas que se mostram mais úteis para a distinção de A. lituratus e A. fimbriatus são o formato do rostro e o grau de desenvolvimento das cristas supra-orbitais e pós-orbitais e dos processos pré-orbitais e pós-orbitais. Somam-se a estas características as dimensões cranianas já citadas, principalmente a largura da constrição pós-orbital, que é visivelmente mais acentuada em $A$. lituratus. 
Myers \& Wetzel (1983), HANDley (1989) e MARQues-AgUiar (1994) trabalharam com exemplares de $A$. lituratus e de $A$. fimbriatus de diferentes pontos de suas distribuições geográficas. Estes autores citam como características diagnósticas cranianas as mesmas observadas nos exemplares examinados provenientes do Rio Grande do Sul. Este fato leva a crer que estas espécies não tenham variações geográficas cranianas acentuadas e que as características diagnósticas citadas são válidas para toda sua área de distribuição.

AGRADECIMENTOS. Ao CNPq pela concessão da bolsa de mestrado (contrato número 83.0350/94-2), que possibilitou a realização deste trabalho. À Fundação Zoobotânica do Rio Grande do Sul e ao Museu de Ciências e Tecnologia da Pontifícia Universidade Católica do Rio Grande do Sul pelo empréstimo de exemplares de suas coleções científicas. Ao Dr. Valdir Antônio Taddei pela confirmação da identificação de exemplares de Artibeus.

\section{REFERÊNCIAS BIBLIOGRÁFICAS}

BARqueZ, R.M. \& R.A. OJeDA. 1992. The bats (Mammalia: Chiroptera) of the Argentine Chaco. Ann. Carnegie Mus. 61 (3): 239-261.

DAvIS, W.B. 1984. Review of the large fruit-eating bats of the Artibeus "lituratus" complex. (Chiroptera: Phyllostomidae) in Middle América. Occ. Pap. Mus. Texas Tech Univ. 93: 1-16.

HANDLEY JR., C.O. 1987. New species of mammals from northern south America: fruit-eating bats, genus Artibeus Leach. Fieldiana Zool., new. ser., 39: 163-172. . 1989. The Artibeus of Gray 1838, p.443-468. In: K.H. REDFORD \& J.F. EISENBERG (Eds). Advances in Neotropical Mammalogy. Gainesville, The Sandhill Crane Press, 614p.

1991. The identity of Phyllostoma planirostre Spix, 1823 (Chiroptera: Stenodermatinae). Bull. Amer. Mus. Nat. Hist. 206: 12-17.

IHERING, H. VON. 1895. Os mamíferos do Rio Grande do Sul. Anuário do Rio Grande do Sul, Porto Alegre, p. 41-77.

JONES, J.K. \& D.C. CARTER. 1976. Annotated checklist, with keys to subfamilies and genera, p.7-38. In: R.J. BAKER; J.K. JONES JR. \& D.C. CARTER (Eds). Biology of Bats of the New World Family Phyllostomatidae. Part I. Texas, Texas Tech University, 218p.

KoopMAN, K.F. 1982. Biogeography of the bats of South America, p.273-302. In:

M.A. MARES \& H.H. GENOWAYS (Eds). Mammalian Biology in South America. Special Publication Series Pymatuning Laboratory of Ecology, University of Pittsburgh, Vol. 6, 539p.

MARQUES-AGUIAR, S.A. 1994. A systematic review of the large species of Artibeus

Leach, 1821 (Mammalia: Chiroptera) with some phylogenetic inferences. Bol.

Mus. Para. Emílio Goeldi, sér. Zool., 10 (1): 3-83.

MYERS, P. \& R.M. WETZEL. 1979. New records of mammals from Paraguay. Jour. Mamm. 60 (3): 638-641.

1983. Systematics and zoogeography of the bats of the Chaco Boreal.

Miscell. Publ. Mus. Zool. Univ. Michigan. 165: 1-59.

OwEN, R.D. 1987. Phylogenetic analyses of the bat subfamily Stenodermatinae 
(Mammalia: Chiroptera). Special Publ. Mus. Texas Tech Univ. 26: 1-65. RADAM/BRASIL. 1986. Levantamento de Recursos Naturais. Vol 33. Folha SH.

22 Porto Alegre e Parte das Folhas SH. 21 Uruguaiana e SI. 22 Lagoa Mirim.

Rio de Janeiro, Secretaria de Planejamento da Presidência da República, Fundação Instituto Brasileiro de Geografia e Estatística, 791p.

REDForD, K.H. \& J.F. EISENBERG. 1992. Mammals of the Neotropics, The

Southern Cone. Chicago, The University of Chicago Press, Vol. 2, 430p.

SANTOS, A.B. 1978. Identificação e dados bio-ecológicos dos quirópteros do Vale do Rio dos Sinos e arredores. Est. Leopold. 13 (45): 75-127.

TADDEI, V.A. 1979. Phyllostomidae (Chiroptera) do Norte-Ocidental do Estado de São Paulo. III - Stenodermatinae. Ciência e Cultura 31 (8): 900-914.

VIEIRA, C.O.C. 1942. Ensaio monográfico sobre os quirópteros do Brasil. Arq. Zool. Est. São Paulo. 3 (8): 219-471.

VIzOTTO, L.D. \& V.A. TADDEI. 1973. Chave para determinação de quirópteros brasileiros. Boletim de Ciências, Faculdade de Filosofia Ciências e Letras, São José do Rio Preto 1: 1-72.

Wallauer, J.P. \& E.P. AlbuQuerQue. 1986. Lista preliminar dos mamíferos observados no Parque Florestal Estadual do Turvo, Tenente Portela, Rio Grande do Sul, Brasil. Roessléria, Belém, 8 (2): 179-185.

Recebido em 10.X.1997; aceito em 07.IV.1999 